\title{
Structure Related Anomalous Fluctuation of Refractive Index and Oxygen Effect in Human Blood Model Saline Water
}

\author{
Shizuo Fujiwara, Fumihiro Arakawa and Osamu ToI \\ Department of Chemistry, Faculty of Science, Chiba University, Yayoi-cho, Chiba 260 \\ S.F.: Present address Research Institute for Information and Knowledge, Kanagawa University, \\ Rokkakubashi, Yokohama 221. All correspondence should be addressed to.
}

\begin{abstract}
An anomalous fluctuation of the refractive index has been observed in the aqueous sodium chloride solution of about $0.1 \mathrm{~mol} / 1 \mathrm{NaCl}$. The latter value corresponds to the salt concentration of the human blood. The fluctuation is just the same as that in the former experiment on the solution of $0.55 \mathrm{~mol} / \mathrm{l}$, which corresponds to the salt concentration of seawater. For the elucidation of the source of the fluctuation, measurements have been made on the mixtures of the heavy water and the normal water, where a similar anomalous fluctuation has been found to occur at the concentration in the range from 15 to $20 \%$ by volume. This finding and the results of the quantum chemical calculation allow the fluctuation of the $0.1 \mathrm{~mol} / \mathrm{l} \mathrm{NaCl}$ solution to be attributed to the fact that the concentration produces an interface of two structurally different phases for the solution. It is noted that the anomalous fluctuations are not observed when the dissolved oxygen is removed from the solutions.
\end{abstract}

Keywords Refractive index, hydrogen bonding, water cluster, human blood, oxygen effect

Analysis of the structure of aqueous electrolyte solutions is one of the important targets of analytical sciences. $^{1}$ Various methods have been used so far for the investigation of the problem. For example, X-ray spectroscopy has revealed the structure of some electrolyte solutions ${ }^{2}$ and C-13 NMR has identified the species being produced in propylene carbonate and water mixtures. ${ }^{3}$ Recently, one of the present authors (S.F.) has shown that an anomalously heavy fluctuation is observed in the refractive index of the aqueous strong electrolyte solutions at a specific concentration. ${ }^{4}$ The latter value varies depending on the species of the salt. More detailed investigation has revealed not only the value of the specific concentration but also the effect of the dissolved oxygen and of the interval of the sampling of data. ${ }^{5}$

Quantum chemical calculations for the thermodynamical energy of the aqueous sodium chloride solution have elucidated the basis of the specific concentration cited above. ${ }^{6}$ The results suggest that the solution of the specific concentration refers to an interface of two different phases, one which is dominated by the free solvent molecules of water and the other by the solvated ions.

A solution whose concentration is lower than the specific concentration thus may be a situation where the ions are dispersed in the bulk media of the solution, whereas a solution whose concentration is higher may be one where the solution can be taken as equivalent to a pile-up of the solvated ions. The point of the problem is that the thermodynamical nature of the solution is divided into two phases by the critical concentration: one phase mainly depends on the dispersion entropy and the other on the internal energy of the solution as a whole.

The data of the heats of infinite dilution of the strong electrolyte solution present clear-cut support for the latter seeing. ${ }^{7}$. More importantly, the value of the specific concentration for sodium chloride as cited above corresponds to the concentration of salt of seawater.

Thus, the authors has taken interest to examine the fluctuation of the refractive index of the sodium chloride solution in the range around $0.1 \mathrm{~mol} / 1$ in order to see if the salinity of human blood carries features analogous to those of the seawater. This paper will report the results of investigation: a similar anomalous fluctuation has been found, and the value of $0.1 \mathrm{~mol} / 1$ $\mathrm{NaCl}$ is another critical concentration which makes an interface in the solution. Again, the anomalous fluctuation ceases when the dissolved oxygen is removed from the solution. The mechanism of this effect of oxygen is not yet elucidated. 


\section{Experimental}

The fluctuations of the refractive index were measured in the same way as that of the previous investigation ${ }^{5}$ except that the output of the refractive index meter was directly sampled and processed by a personal computer PC-9801, Nippon Electric Company. The output of the meter, which actually refers to the difference of the refractive indices of the sample and the reference solutions, is sampled for $1 \mathrm{~min}$ at an intervals of $1 \mathrm{~ms}$. Six thousand data are thus obtained with one measurement run. They are then broken into groups of data of different sampling intervals. Group No. 1 refers to the data with an interval of $1 \mathrm{~ms}$, No. 2 for the intervals of $2 \mathrm{~ms}$, No. 3 for those of $3 \mathrm{~ms}$ and so on. Statistical analysis of the data for each of the groups was carried out by the computer. The fluctuation, $\bar{\sigma}$, has also been calculated for each group in the same way as that in the former investigation. ${ }^{5}$

The aqueous sodium chloride solutions were prepared by dissolving the analytical grade sodium chloride of the Wako Pure Chemical Industries with a concentration difference of $0.01 \mathrm{~mol} / \mathrm{l}$.

We wanted to see how the hydrogen bonding influenced the structure of the electrolyte solution, so measurements of $\bar{\sigma}$ were also made on the mixtures of the heavy water and the normal water. We assumed that the hydrogen bonding in the normal water was controlled by the heavy water resulting in a change of the refractive index of the water. $2.5 \%$ by volume of heavy water was added to the normal water.

\section{Results and Discussion}

The results of measurement for $\mathrm{D}_{2} \mathrm{O} / \mathrm{H}_{2} \mathrm{O}$ are shown in Fig. 1 , where the concentration of $\mathrm{D}_{2} \mathrm{O}$ is taken along the $x$ axis, the sampling time interval along $y$ and the fluctuation of the refractive index, $\bar{\sigma}$, along $z$. The figure presents a lineup of $\bar{\sigma}$ 's of all groups of data. The front most line in the figure refers to the fluctuation of group No. 1 for the data of $1 \mathrm{~ms}$ sampling interval, the next line to that of group No. 2 and so on.

It is obvious in the figure that all lines show a peak at the concentration of $15 \% \mathrm{D}_{2} \mathrm{O}$ and fall to the ground level at $20 \%$.

Figure 2 presents the results of the sodium chloride solutions which exhibit a drastic drop of $\bar{\sigma}$ at the concentration range from 0.03 to 0.10 and almost a zero value as the average for the higher concentration range. Again, the $x$ axis in Fig. 2 refers to the concentration of salt, $y$ to the sampling time interval and $z$ to the fluctuation, $\bar{\sigma}$.

The results of Fig. 1 on the $\mathrm{D}_{2} \mathrm{O} / \mathrm{H}_{2} \mathrm{O}$ system suggest that an anomalously heavy fluctuation of the refractive index occurs in the system of $15-20 \% \mathrm{D}_{2} \mathrm{O}$, which may imply the formula of the system of $\mathrm{D}_{2} \mathrm{O}\left(\mathrm{H}_{2} \mathrm{O}\right)_{4}$.
Actually, a quantum chemical calculation has shown recently that the tetrahedral coordination is the most probable arrangement of the cluster structure of water. ${ }^{8}$ Raman spectroscopists have reported that the cluster structure is one possible form for the interpretation of the spectra. ${ }^{9}$

These results suggest a possible basis for the interpretation of the fluctuations of the aqueous sodium chloride solutions. It is normal that electrolytic ions which are dissolved in water break the hydrogen bonding of the water. The chemical shift of the proton NMR in polar liquid is usually interpreted in terms of the formation and destruction of the hydrogen bonding. ${ }^{10}$ If this is the case with the aqueous sodium chloride solution, the hydrogen-bonded structure of water may be broken by the introduction of the ions, which must result in the formation of the cluster of water molecules. As in the case of the $\mathrm{D}_{2} \mathrm{O} / \mathrm{H}_{2} \mathrm{O}$

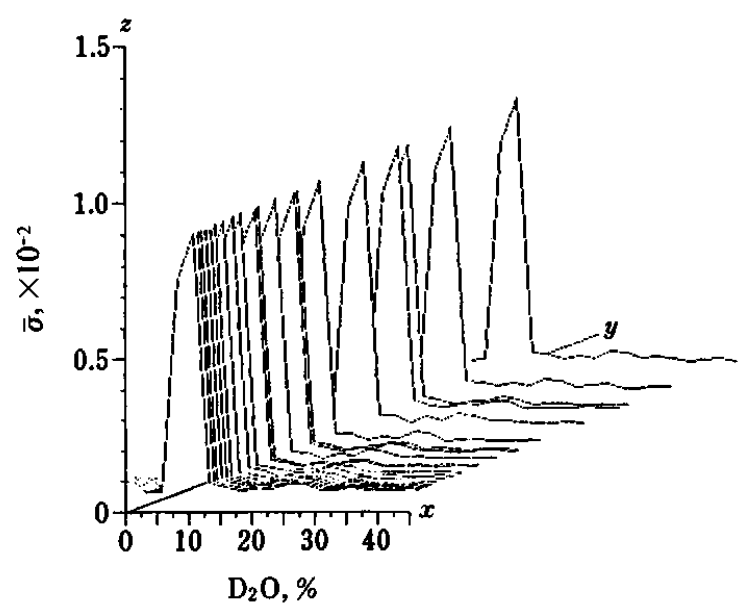

Fig. 1 Fluctuation of the refractive index, $\bar{\sigma}$, of $\mathrm{D}_{2} \mathrm{O}-\mathrm{H}_{2} \mathrm{O}$ mixtures. $x$, concentration of $\mathrm{D}_{2} \mathrm{O} \%$ /volume. $y$, interval of data sampling in ms. $z$, fluctuation. The front most line referes to $\bar{\sigma}$ for $1 \mathrm{~ms}$ of $y$, the next line for $2 \mathrm{~ms}$, and so on.

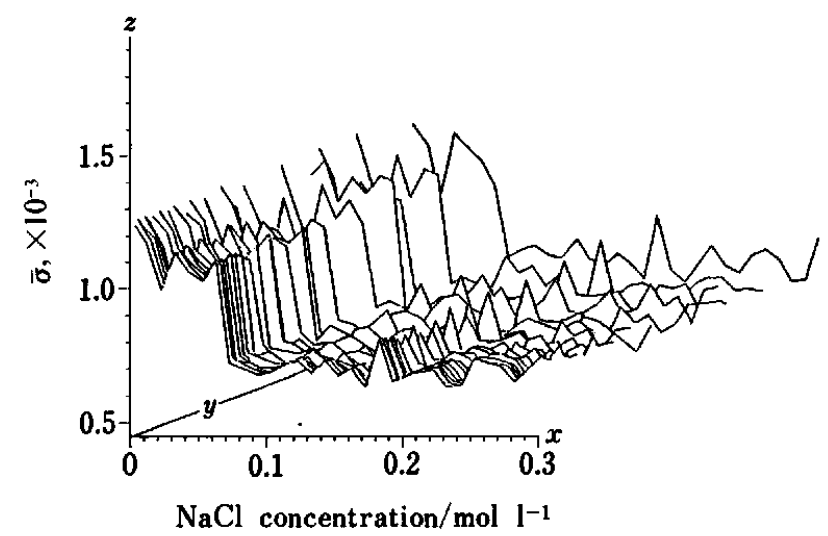

Fig. 2 Fluctuation of the refractive index, $\bar{\sigma}$, of the aqueous sodium chloride solution. $x$, concentration, $\mathrm{mol} / \mathrm{NaCl} . y$, interval of data sampling in ms. $z$, fluctuation. 
system, the aqueous sodium chloride solution must start with the competition between the two phases of water, one dominated by the unified system of the hydrogen bonded water molecules and the other governed by the clusters.

The specific concentration of $0.1 \mathrm{~mol} / 1 \mathrm{NaCl}$ may be the critical concentration for the competitive two phases, and accordingly, may cause the anomalous fluctuations of the refractive index. The situation would be just the same as that in the case of the seawater. The only difference between the two is that the key of the critical work for the seawater is the hydration of ions, whereas that for the human blood is the hydrogen bonding. Both cases may rely on the nature of the solution at the critical interface of the dispersion entropy of the solute individuals and the internal energy of the solution. ${ }^{1,7}$ This remark must play an important role in the sea and in the human body.

The fact that the anomalous fluctuation ceases when oxygen is removed from the solutions of $0.1,0.5 \mathrm{~mol} / \mathrm{l}$ $\mathrm{NaCl}$ and $20 \% \mathrm{D}_{2} \mathrm{O}$ must also play an important role in nature. All these problems await further investigation.
Authors' thanks are due to Prof. B. M. Rode of the University of Innsbruck for valuable discussions.

\section{References}

1. S. Fujiwara, Anal. Sci., 1, 306 (1985).

2. H. Ohtaki, Reviews Inorg. Chem., 4, 103 (1982).

3. S. Fujiwara and Y. Nishimoto, Spectrosc. Lett., 17, 633 (1984).

4. S. Fujiwara and Y. Nishimoto, Proc. Japan Academy, 59, B, 333 (1983).

5. S. Fujiwara, Y. Nishimoto and F. Arakawa, Anal. Sci., 1, 23 (1985).

6. J. P. Limtrakul, S. Fujiwara and B. M. Rode, Anal. Sci., 1, 29 (1985).

7. S. Fujiwara, Geochem. J., 13, 225 (1979).

8. G. C. Lie and E. Clementi, IBM Technical Report, KGN-36 (1985).

9. G. E. Walrafen, J. Chem. Phys., 47, 114 (1967); 48, 244 (1968); 50, 560, 567 (1969).

10. J. A. Pople, W. G. Schneider and H. J. Bernstein, "High Resolution Nuclear Magnetic Resonance", p. 400, McGrawHill Book Co., Inc., New York (1959). 